Article -Agriculture, Agribusiness and Biotechnology

\title{
Hydraulic System Onboard Monitoring and Fault Diagnostic in Agricultural Machine
}

\section{Saurabh Gupta ${ }^{1}$}

https://orcid.org/0000-0003-1892-1732

Mahdi Khosravy ${ }^{2,3^{*}}$

https://orcid.org/0000-0001-5375-391X

\section{Neeraj Gupta ${ }^{4}$}

https://orcid.org/0000-0003-4634-547X

Hemant Darbari ${ }^{1}$

https://orcid.org/0000-0002-0730-781X

\author{
Nilesh Patel ${ }^{4}$ \\ https://orcid.org/0000-0001-7543-8590
}

${ }^{1}$ John Deere India PVT. LTD., Department of Advanced Engineering, Pune, India; ${ }^{2}$ Federal University of Juiz de Fora, Electrical Engineering Department, Juiz de Fora, MG, Brazil; ${ }^{3}$ University of the Ryukyus, Electrical Engineering Department, Okinawa, Japan; ${ }^{4}$ Oakland University, Rochester City, Michigan, USA

Received: 2018.07.17; Accepted: 2019.05.19

*Correspondence: mahdi.khosravy@ufjf.edu.br; Tel.:+55-32-991674776 (M.K.)

\section{HIGHLIGHTS}

- Onboard automatic fault diagnosis for agricultural machinery hydraulic systems.

- A low computational burden pseudo-spectrum analysis of oil filter sound tracks.

- Implementation by the cellphones microphones and recorders of three different commercial brands.

- Introducing efficient features in MUSIC pseudo-spectrum of cellphone records of oil filter sound.

Abstract: Agricultural Machinery as an off-road vehicle is the backbone of the World agricultural industry. Its main function is to operate as a prime mover and support the power requirements to function the various type of draft implements. In this regards, the hydraulic system is an important part and is controlled by the propagated oil which is cleaned by impurities and debris using a filter system. Once it blocks, the bypass opens to avoid any pressure burst of the system, and the particles find their way into the hydraulic system and get lodged in the gears, pumps, valves, and drive train to hinder the performance of the Agricultural Machinery. This research presents an onboard Multiple Signal Classification Algorithm (MUSIC) and pseudo-spectrum analysis as a computational tool used by cellphones to analyze the particle pollution level of the hydraulic filter. This analysis is carried out on the soundtracks recorded 
from different cell phones in different incremental stages of fluid contamination to the particles until it being choked, based on the standard of ISO4406.

Keywords: Predictive maintenance; preventive maintenance; hydraulic systems; acoustic measurements; computerized instrumentation; acoustic signal detection; fault diagnosis.

\section{INTRODUCTION}

The agriculture industry is suffering from labour scarcity and uncertainty of rains [1]. The report [2] from Food and Agriculture Organization of The United Nations sheds some light on the nature of the challenges that agriculture and food systems are facing now and throughout the 21st century and provides some insights as to what is at stake and what needs to be done. There will be 9.7 billion people on this earth by 2050 . Two third of the people will live in urban areas where demand for the food is grown without expanding agriculture lands. To meet the food requirements of the population, there is a need for autonomous AgMachine (e.g. combines harvesters, crop care etc.). Autonomous farm machines will help us to improve productivity, reduction in time and production cost for farmers even in odd weather conditions. To make autonomous farm machinery successful with the reduction in downtime, an onboard diagnosis system is required in a big way [3]. SAE and many experts Bryant Walker Smith, the professor at the University Of South Carolina School of Law and School of Engineering and one of the top experts in the world of the driverless car have described six levels of autonomy [4]. With increasing levels from level zero to level five, the kind of autonomy is possible through a precise fault diagnostic computational system.

Inspired by the development of automotive industry there will be a need for the robust computational system to record onboard monitor of health condition to timely predict the required maintenance and avert potential hazardous condition. This onboard diagnostic system will communicate the present status of the AgMachine system on satellite networks instead of raw data, which can predict the operational command directly. Fig. 1 shows the context diagram for autonomous AgMachine. An interaction of AgMachine with GPS system (1), telemetry system/cellular network (2) for guidance and communications, and data storage and processing units (4) is shown, where (3) presents major subsystem on the AgMachine. The electronic system not only controls the engine, transmission, steering and other precision farming systems; also sends and receives the data on nets. Current electronics system is capable of capturing the real-time health and performance of the AgMachine aggregates and transmitting data through the cellular network using a cellular modem.

Implementation of a hardware device to be installed with AgMachine may be one solution where the use of sensors increases the cost of AgMachine and complexity of the data analysis in autonomous farm machinery system. Associated cost analysis hinders to install above device to AgMachine system for condition monitoring of all the aggregates. For satisfying following conflicting objectives of stakeholders, we need economic and easy to use fault diagnosis and monitoring system: 


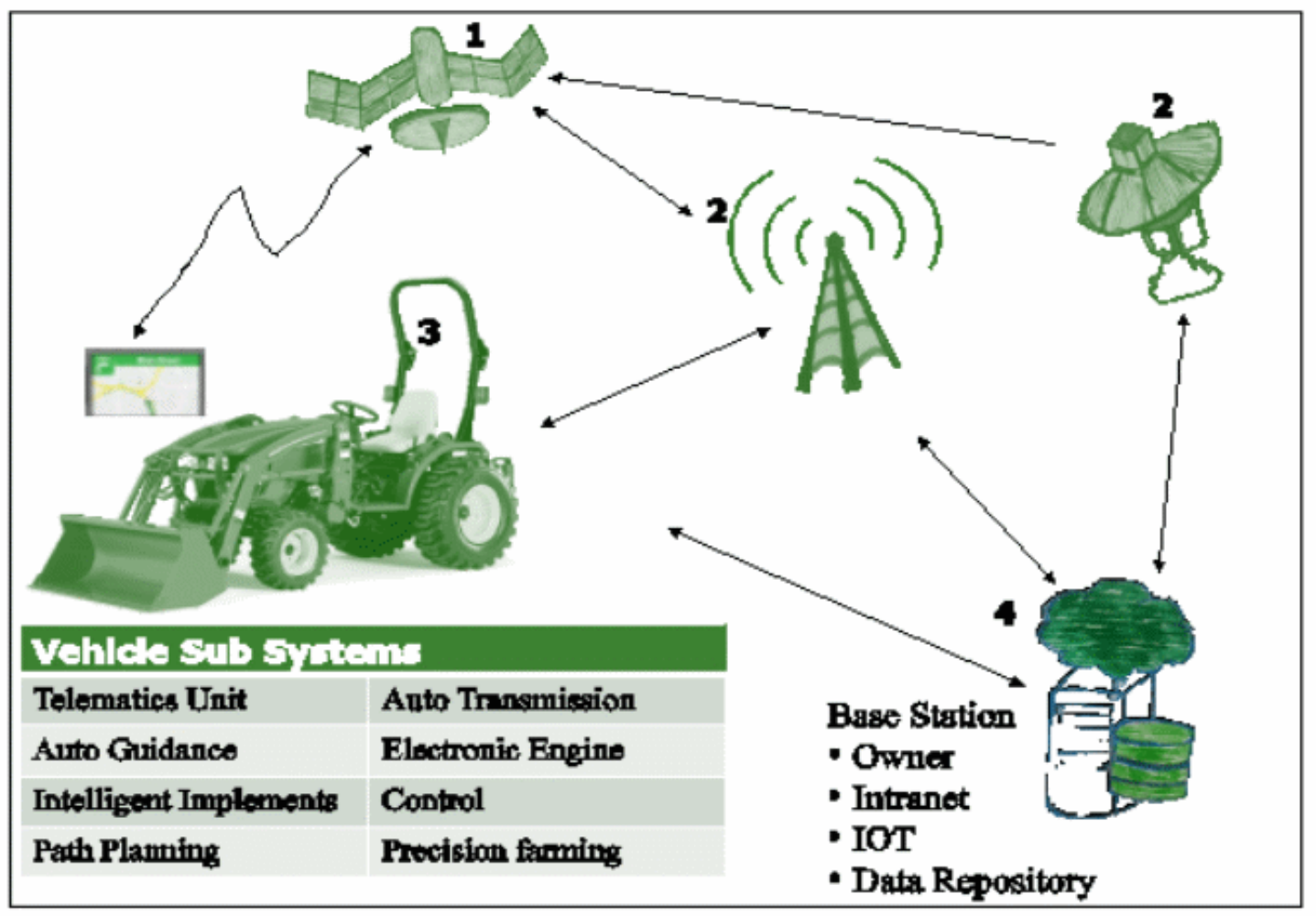

Figure 1. Context diagram of autonomous AgMachi

1. Dealership staff skills/costs need to be balanced.

2. Access must be easy for less technical staff at dealerships.

3. Vehicles need new economic and easy to understand technology and features to attract buyers.

Present oil filter monitoring: Although fruitful research has been performed on prognostics of mechanical and rotational equipment [5-8], there are few reports for predictive maintenance of hydraulic system parts of other vehicles. In an AgMachine case, the hydraulic system is subjected to regular maintenance due to heavy working load. Namely, oil filter guards the system components from destruction resulted from the fluid pollution to the particles. As Damen Technical Agencies [9] reported, in average every minute, around one million particles with the size more significant than one micron $(>1 \mu \mathrm{m})$ enter a hydraulic system which can severely damage the components of the system, and result in costly tribological failure of the system [10]. The Required Cleanliness Level (RCL) [11] is the upper bound for the concentration of particles in a hydraulic system. As contamination passes the RCL, the costly cleaning operations are imposed on hydrological operators. Cleanliness Monitoring provides the timely on time warning of the problems mentioned above for the appropriate maintenance of oil filter, and thus a considerable increase in lifetime of the hydraulic system.

Three modes of particles monitoring are suggested in the literature as off-line, online and in-line which are respectively from a fluid sample, flow line, and pressurized line [12]. Off-line monitoring suffers from the need of expert operator skills for sampling and analysis. This system is existing right now for fault diagnosis of the AgMachine subsystems. It is due to the need for extraction of fluid for analysis in a laboratory. Furthermore, each monitoring results in time extension, and in the result of sampling and analysis, additional pollution interferes the effects and leads to the error in data. In both online and in-line monitoring, either automatic particle counters (APCs)[13] are installed in connection with the flow, or filter blockage monitors (FBMs)[14] are in use of on-road vehicles. APCs work based on the principle of light extinction where a narrow a 
light beam illuminates passage of fluid, and the polluting particles obscure the light beam by blockage or scattering. Thereof corresponding electrical pulses are produced which their number and amplitude determine the size and density of the particles. The disadvantage of deployment of APCs is their high sensitivity to the optical interfaces such as air bubbles, water droplets, additive silicone anti-foam droplets, emulsions, etc. which result in erroneous data and wrong conclusions and thus waste maintenance [15]. Ref. [4] explains the same pain with increasing electronics controls on farm machinery. Twenty technologies are tending to change agriculture system, Ref. [16] describes the need for onboard computation power, e.g. telematics, soil and crop sensors, Mini-chromosome technology, Farm future daily, BUS technology; hyperprecision, Automated grain off-loading; Pervasive automation; Electric drive systems; Autonomous vehicles; 4-G Networks, mobile computing, Internet of things to say few. Based on the above discussion it is prominent that selection or designing of onboard fault diagnostics systems with high computing power shall be able to fill the identified gaps:

1) There is no specific method of recognizing the complete blockage of the hydraulic filter. The operator has to guess and constantly keep a check on the filter to know when to replace it. Although the manufacturer claims the filter to be useful for 300 hours, conditions in the field may increase or decrease this lifespan. There is no method to alert the operator of this change. This can lead to early or delayed change, both of which are expensive and inefficient.

2) When it comes to vibration analysis, the pumps operating at lower speeds either give improper values or values that are very similar to the different qualities of the pump. There is no specific method to gain an accurate answer for this.

It would be worth to say that the proposal demonstrated in this paper can be implemented with another sub-system of the AgMachine with the same mechanism. It is not limited to only filter system of the vehicle.

\section{DEVELOPING FAULT DIAGNOSTIC TOOL}

Nowadays, smartphones have an increasing impact on society in moving toward higher convenience through more and more practical applications. As discussed above that Using any electro-mechanical monitoring system to integrate with the AgMachine is uneconomic and bulkier compared to the developing an application for the smartphone, which is available approximately in every hand, nowadays. In complex and uncertain operating state, proposed technology could be helpful in monitoring and detection of the operational condition of the AgMachine in a field far away from a possible workshop, especially during the emergency circumstances. Flip-side of this proposal will save unnecessary visits to the workshop and expert services as for a routine checkup, which is not always possible and even it turns out to be uneconomic and time-consuming. This can solely be resolved by making a new monitoring and diagnostic system by using an app-based mobile application with the subsequent concept. Thus, it is proposed to use the smartphone to capture the unwanted noise sound signal of the aggregates on the AgMachine and use the computational method to detect the current health of the particular aggregates using in hand smartphone device instead of transferring voluminous data to the data-centres. This scheme with the present communication techniques is described in the Fig. 2. 


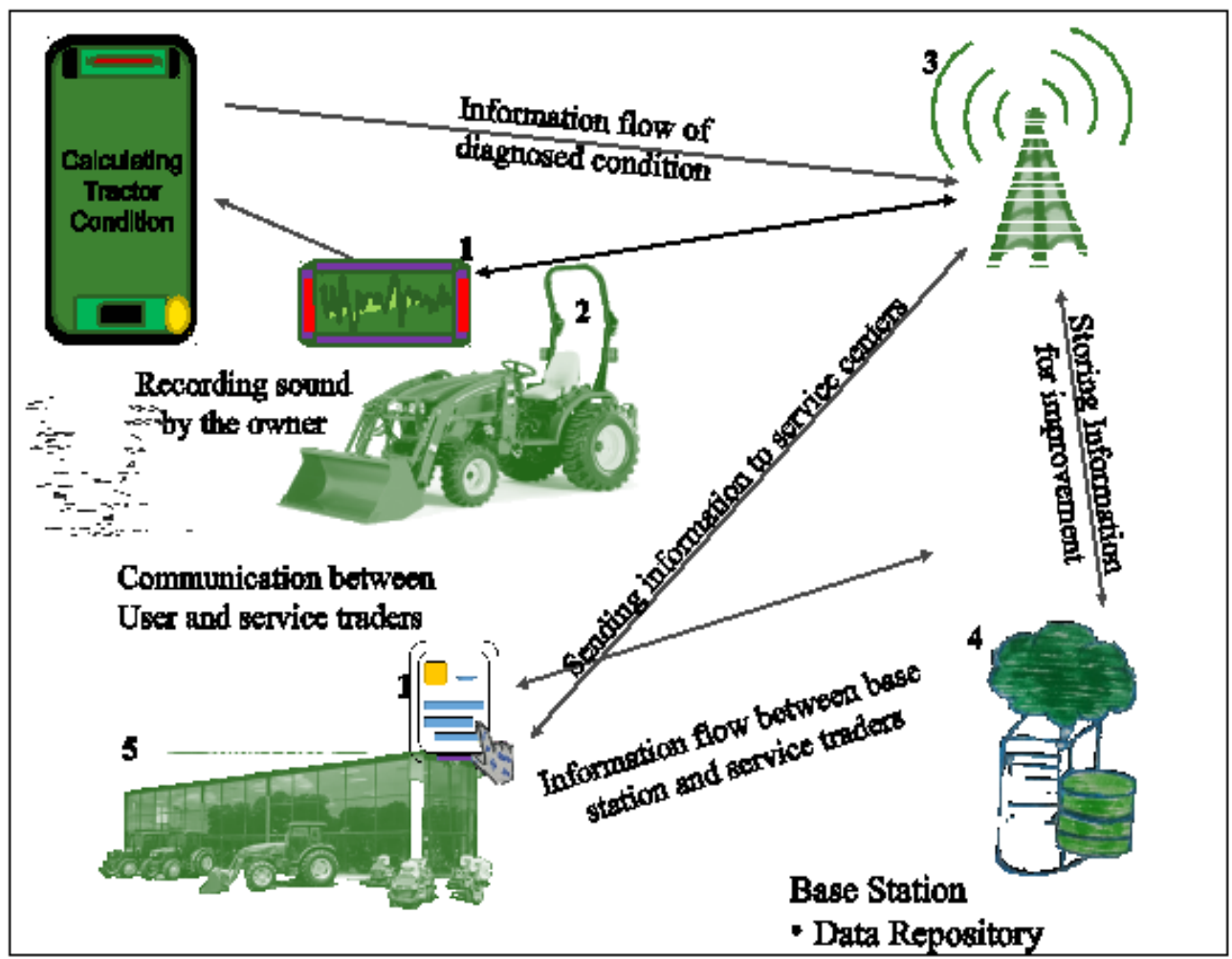

Figure 2. Proposal for fault diagnosis system.

Users may swiftly record the sound and detect the output by the diagnostic algorithm, and it can be transmitted on the net very fast instead of raw data to the base station, dealers and service centers to avoid any severe damage, delay in diagnosis and down of machine with quick and valuable services. Moreover, when a AgMachine works on the field, minor faults in filter system may be the cause of cascading failure, which may not appear before the complete blackout of the full system. Portable and hand in use monitoring and diagnosis system with the precise computational method in complex and increasingly uncertain operating conditions provides an improved facility to the users to in order to measure the status of the AgMachine and thereof increasing the working efficiency. Here, the pseudo-spectrum of oil filter soundtracks is studied and analyzed for the on-line monitoring and prognostics of the hydraulic fluid pollution condition. A preprocessing stage can be applied to the soundtracks before handing to the algorithm [17-21]. To separate the environmental noise background from the content blind component processing methods [22] blind source separation [23-26] and principal component analysis [27] can be additionally augmented to remove the unwanted component by noise canceller filters [28].

An approach for detection of particle pollution level as well prediction and diagnosis of hydraulic choking are introduced. By integrating the proposed smart diagnostic algorithm with cellphones as maintenance and diagnostic application to the AgMachine, even a non-expert operator can monitor its hydraulic condition by just locating the phone in a proper place adjacent to the oil filter. It does not need any requirement of technical skills and the knowledge of the mechanical technology of the particular AgMachine operation to the users. Thus, the proposal offers a demanding tool as an intelligent system to the customers with respect to increase accuracy in fault detection. Since the proposed approach deploys the hydraulic filter sound noise, it directly connects to its activity and is not susceptible to interfering error such as optical errors in APCs. It would be worth to discuss again that it does not need expensive 
optical equipment, as well does not require any installation and related calibrations. It is an approach to realize a user-friendly smartphone diagnostic application.

\section{Data collection}

First and the most important part of the research work over AgMachine filter fault diagnosis is data collection. In our research work, the experiment is done on AgMachine oil filter having the following specification for condition and monitoring through the audio signal records by three different mobile phones (i) Filter Rating is 10 microns (ii) Filter Efficiency (beta efficiency)known as beta 10 is $751 \%$. (iii) Flow rating through the filter is $60 \mathrm{lpm}$, and (iv) Operating pressure to flow the oil through the filter is 200 bar.

The experimental setup is given in Fig. 3, which primarily include a pump, motor, sump, hydraulic filter and suction valves to mimic the flow in the hydraulic system of the AgMachine. The data will be collected in the form of sound noise with the help of microphones to create a database that contains the sound of various stages of the operations of pump filters. The first step is to capture the noise of the environment, without the setup. This will help us create an identity to the noise sound that has to be filtered out by the analysis software. Next, a fresh batch of oil is introduced into the sump. The setup is turned on. After 30 seconds (ideal stabilization time), the noise is recorded again. Post this, and we add a certain amount of sawdust to the sump to mimic the field conditions. 30 seconds are given to the pump to idealize its functioning after adding particulate, then the noise is recorded. This process is repeated until the bypass valve is activated.

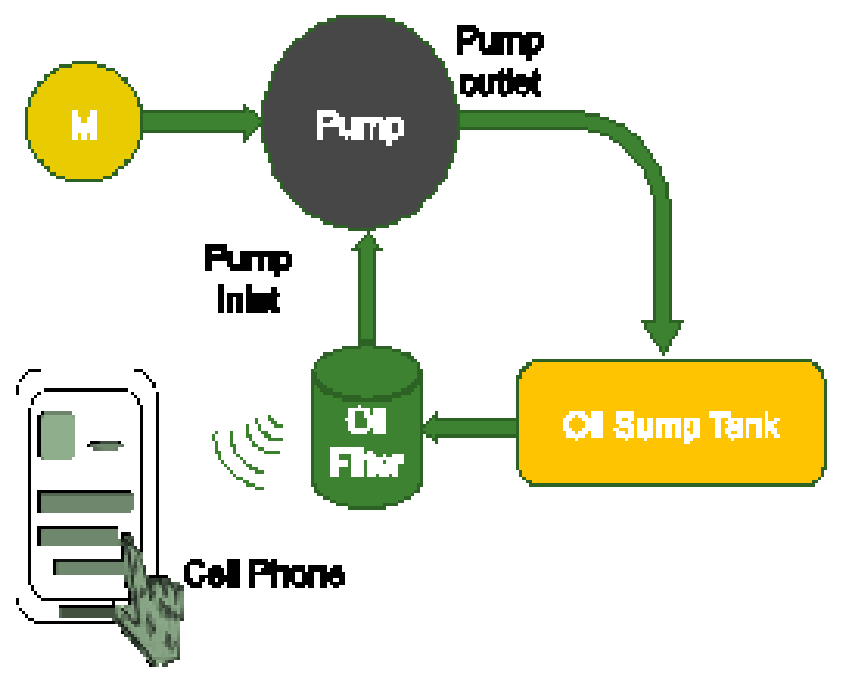

Figure 3. Schematic setup for recording oil filter sound by smart-phone.

This process is repeated for filters of different ages and usage times to create an extensive database for noise matching. Analyzing noise-free dataset is easier and more efficient, the data with noisy background better matches the realistic field environment. Although providing multi-sensory data can be more useful for data processing, the fault diagnosis through single sensory data records of each part is a valuable task in increment of the user-friendly quality of the mobile phones application. In order to promote the user-friendly utility of the application, single-sensory sound records are acquired. Despite the fact that of the higher efficiency of feature extraction from the multi-sensory data, it requires a sophisticated microphones arrangement, while single-sensory records can be obtained merely by putting the smartphone in its 
prearranged location adjacent to oil filter as shown in Fig 3. On the other side, multisensory data not only brings the advantage of easing pre-processing of the data and removal of noise and signals interference, but also it provides more features and more accurate diagnosis. However, it requires more sophisticated microphones arrangements around the vehicle and therefore the complicated application for the user.

\section{Collection of sound track of oil filter}

To develop the study over processing of each sensor data in connection to its related part, availability of the single sensory signal with an acceptable level signal to interference ratio (SIR) is an essence. Considering that each defect has its individual generated vibration sound signal produced by the defected part, and since one part can have different levels of fault, its associated defect vibration signal covers a broad class of defect indicating signals. So, in general, each assumed part, can have two types of possible vibration signals; the vibration signal caused by ageing and the vibration caused by defect or damage. This can also be discriminated by the proposed methodology and can be extended to any faults and for any subsystem of the AgMachine. The general methodology of single sensory data collection deployed by the authors is as follows:

First, we have recorded the ageing-related sound signals by increasing the particle pollution in the hydraulic system which directly affects the ageing stage of the filter, and after that, failure concerning signals. Since, we are going to have an ideal signal free of any interference, which is an oil filter with the age of $T_{A}$ is mounted on a new AgMachine with new parts and calm engine. Apart from a possible defect problem with its generated vibration sound, ageing and pollution produce their corresponding vibration signals that can be categorized under a class of signals for different levels of ageing. To avoid the other parts vibration fault signals interference and to have a clear view of the concerning failure, the experiments are done on a new tractor hydraulic system. The sound recorder device is mounted in proper place, and the sound noise of the component is recorded.

Here, in recording the soundtracks of the oil filter, just the usual condition of the agricultural machinery with a regular engine rotation per minute (RPM) of 900 . The recorded data is related to the different levels of particles pollution inside the hydraulic system. These levels are arranged based on ISO4406 [29] from zero which means new oil filter and clean fluid, one level, two levels and so on till six-levels of polluting particles. After that, the oil filter is choked that is the last level of audio recording. This classification is arranged in consultation with service technicians based on the standard mentioned above. Note that, the age of a part is not the matter of time but the efficacy of the section as here it is categorized into three or five age groups as new, young, middle age, old and very old. This age classification is also considered in consultation with agriculture machine service technicians. The methodology mentioned above and classification for the collection of single sensory data is just a simplified explanation of what we have done in laboratories. In this research work, the practice is performed on tractor oil filter fault diagnosis through audio tracks recorded by three different popular brands of cell phones. Here to avoid mentioning the name of the brands, we just call them as first, second and third cellphones.

\section{Pseudo-spectrum analysis}

After collection of single sensory noise audio signal records by three different mobile phones, we process the audio tracks in connection with the density level of polluting particles. Sound classification based on features is a fundamental research [30].The level of the dust inside the pump determines the age of the pump before being clogged. In order to detect the clogging stage and predict being choked with particles, a precise feature should be chosen. To aim this goal, here, the pseudo-spectrum 
analysis of the audio tracks is proposed. Here, a brief review to pseudo-spectrum is given.

1) Pseudo-spectrum: A well-behaved function: $f(x)$, in an interval, can be represented as expansion in a set of orthonormal functions, $P_{n}(x)$, as follows:

$f(x)=\sum_{n=0}^{\infty} a_{n} P_{n}(x) \quad x \in[a, b]$

where $P_{n}(x)$ 's are orthonormal polynomials as:

$\int_{a}^{b} \omega(x) P_{n}(x) P_{m}(x) d x= \begin{cases}1, & m=n \\ 0, & m \neq n\end{cases}$

where $\omega(x)$ is an appropriate weight function for orthonormal condition. Orthonormal polynomials as basis functions are classically well known as Bernard Shizgal has listed some of them [28]. An approach to pseudo-spectrum is eigenvector method. The pseudo-spectral method can be provided by eigenvalues as $\int_{a}^{b} k(x, y) \psi_{n}(y) d y=\lambda_{n} \psi_{n}(x)$.

2) Pseudo-Spectrum by Multiple Signal Classification: We are looking for an estimation of a set of reliable constant features/parameters to which the audio records are dependent. There have been some classical methods fulfilling the same goal, such as maximum likelihood (ML) [32] and maximum entropy (ME) [33]. However, the aforementioned classical methods suffer from parameters estimation sensitivity and bias of the parameters. These problems were overcome by the deployment of the covariance matrix of the signal for estimation of complex sinusoids by Pisarenko [34]. Later, Schmidt by deriving a geometric solution developed multiple signal classification algorithm (MUSIC) [35]. MUSIC estimates pseudo-spectrum of the signal using the estimates of the eigenvectors of the correlation matrix of input data signal. MUSIC estimation of the signal frequency content is by the deployment of eigenspace. Its assumption of the input signal is $p$ complex exponentials together with background noise. The spanned subspace of signal is by the $p$ eigenvectors corresponding to the $p$ largest eigenvalues of $\mathbb{R}_{x}$, the $M \times M$ autocorrelation matrix of the input signal, while the sorting order of eigenvalues is in decreasing direction. In MUSIC pseudo-spectrum, the $M$ dimensional space is decomposed to signal and noise components based on the eigenvectors of correlation matrix. Considering the $M$ vector length $\boldsymbol{x}(n)$ as its current value and the next coming samples $x(n)=[x(n) x(n+1) \ldots x(n+M-1)]^{T}$, then the signal model consisting of complex exponential is as follows:

$\boldsymbol{x}(n)=\sum_{p=1}^{P} \alpha \boldsymbol{v}\left(f_{p}\right) e^{j 2 \pi n f_{p}}+\boldsymbol{w}(n)=\boldsymbol{s}(n)+\boldsymbol{w}(n)$

where $\boldsymbol{w}(n)$ is the noise vector, and $\boldsymbol{v}(f)=\left[1 e^{j 2 \pi f} \ldots e^{j 2 \pi(M-1) f}\right]^{T}$ is the vector of frequency components. Then the autocorrelation of this signal model is as follows:

$\boldsymbol{R}_{x}=\boldsymbol{E}\left\{\boldsymbol{x}(n) \boldsymbol{x}^{H}(n)\right\}=\boldsymbol{R}_{s}+\boldsymbol{R}_{w}$

$=\sum_{p=1}^{P}\left|\alpha_{p}\right|^{2} \boldsymbol{v}\left(f_{p}\right) \boldsymbol{v}^{H}\left(f_{p}\right)+\sigma_{\omega}^{2} \boldsymbol{I}=\boldsymbol{V} \boldsymbol{A} \boldsymbol{V}^{H}+\sigma_{\omega}^{2} \boldsymbol{I}$

$\boldsymbol{V}$ is a $\mathrm{M} \times \mathrm{P}$ matrix whose columns are the frequency vectors in (3), $\boldsymbol{V}=$ $\left[v\left(f_{1}\right) v\left(f_{2}\right) \ldots v\left(f_{P}\right)\right]$. The second term is correlation matrix of white noise $\left(\boldsymbol{R}_{w}=\right.$ $\left.\sigma_{\omega}^{2} I\right)$, and $\boldsymbol{R}_{S}$ is correlation matrix of the signal that is a rank deficient matrix due to $\mathrm{P}<M, \boldsymbol{R}_{w}$ is a full-rank matrix. The autocorrelation matrix $\boldsymbol{R}_{x}$ can be written as eigendecompositio

$\boldsymbol{R}_{\boldsymbol{x}}=\sum_{m=1}^{M} \lambda_{m} q_{m} q_{m}^{H}=\boldsymbol{Q} \boldsymbol{\Lambda} \boldsymbol{Q}^{H}$ 
where $\lambda_{m}$ are eigenvalues in descending order, and $q_{m}$ are the corresponding eigenvectors. $\boldsymbol{\Lambda}$ is a diagonal matrix with the eigenvalue in descending order on the diagonal while the columns of $\boldsymbol{Q}$ are the corresponding eigenvectors. Therefore, the $P$ largest eigenvalues correspond to the signal complex exponential components and the remaining eigenvalues which have equal value correspond to the noise. As a result, we can partition the correlation matrix to two parts, the part due to the signal eigenvectors and the part due to the noise eigenvector

$\boldsymbol{R}_{\boldsymbol{x}}=\boldsymbol{Q}_{s} \boldsymbol{\Lambda}_{s} \boldsymbol{Q}_{s}{ }^{H}+\sigma^{2} \boldsymbol{Q}_{w} \boldsymbol{Q}_{w}{ }^{H}$

Where $\boldsymbol{\Lambda}_{S}$ is a $P \times P$ diagonal matrix of the signal eigenvalues, and $\boldsymbol{Q}_{s}=$ $\left[\begin{array}{llll}\boldsymbol{q}_{1} & \boldsymbol{q}_{2} \ldots & \boldsymbol{q}_{P}\end{array}\right]$ and $\boldsymbol{Q}_{w}=\left[\boldsymbol{p}_{P+1} \ldots \boldsymbol{p}_{M}\right]$ are matrices with columns of the signal and noise eigenvectors, respectively. Therefore, the M-dimensional subspace is split into two subspaces spanned by the signal and noise eigenvectors which are orthogonal to each other since the correlation matrix is Hermitian symmetric. Consequently, we can write the matrices that project an arbitrary vector onto the signal and noise subspaces $\boldsymbol{P}_{S}=$ $\boldsymbol{Q}_{s} \boldsymbol{Q}_{s}{ }^{H}, \boldsymbol{P}_{w}=\boldsymbol{Q}_{\mathrm{w}} \boldsymbol{Q}_{w}{ }^{H}$. The eigenvectors of the correlation matrix are orthonormal $\boldsymbol{Q}_{s}{ }^{H} \boldsymbol{Q}_{s}=\boldsymbol{I}, \boldsymbol{Q}_{w}{ }^{H} \boldsymbol{Q}_{w}=\boldsymbol{I}$, and the two subspaces are orthogonal;

$$
\boldsymbol{P}_{\boldsymbol{s}} \boldsymbol{v}\left(f_{p}\right)=\boldsymbol{v}\left(f_{p}\right), \quad \boldsymbol{P}_{w} \boldsymbol{v}\left(f_{p}\right)=\mathbf{0} .
$$

Despite Pisarenko, MUSIC does not limit the length of the time window to $\mathrm{P}+1$, and allows it to be more than $P+1$.. Consequently, it provides an averaging space longer than one over the noise subspace, thereof it results in an improved and more robust frequency estimation method. Due to the orthogonality between the noise subspace and signal subspace, for each eigenvector

$\boldsymbol{v}^{H}\left(f_{p}\right) \boldsymbol{q}_{m}=\sum_{k=1}^{M} \boldsymbol{q}_{m}(k) e^{-i 2 \pi(k-1) f_{p}}=\mathbf{0} . \quad p \in[1, \ldots, P], q \in[P+1, \ldots, M]$

Thus, for each noise eigenvector, a pseudospectrum is computed as follows

$\overline{R_{m}}(i 2 \pi f)=\frac{1}{\left|\boldsymbol{v}^{H}\left(f_{p}\right) \boldsymbol{q}_{m}\right|^{2}}=\frac{1}{\left|Q_{m}(i 2 \pi f)\right|^{2}}$.

$\mathrm{P}$ of $\mathrm{M}-1$ roots of $\mid Q_{m}(i 2 \pi f)$ correspond to the complex exponentials frequencies. $\mathrm{M}-\mathrm{P}$ noise eigenvectors share these roots where some of them are close to the unit circle and result in extra peaks. MUSIC by averaging M-P pseudospectra of the noise eigenvectors reduces the levels of these peaks:

$\bar{R}_{M U S I C}(i 2 \pi f)=\frac{1}{\sum_{m=P+1}^{M}\left|v^{H}\left(f_{p}\right) \boldsymbol{q}_{m}\right|^{2}}=\frac{1}{\sum_{m=P+1}^{M}\left|Q_{m}(i 2 \pi f)\right|^{2}}$

3) Oil Pump Fault Diagnosis by MUSIC: At this moment application of MUSIC algorithm on each signal record, its pseudo-spectrum is estimated. After that, the pseudo-spectrum curves are compared and evaluated over the frequency range of the signals. Although MUSIC-estimated pseudo spectrum is over normalized frequencies (in rad/sample), by having the sampling frequency of the records, we can perform the evaluation on frequencies in Hertz.

\section{RESULTS AND DISCUSSION}

The MUSIC pseudo-spectrum of each mobile phone sound records corresponding to eight different conditions is analyzed. The audio tracks are recorded under different conditions as follows: 
Track 1 The sound of oil pump just as the engine starts. The hydraulic system is clean, and RPM is 900.

Tracks 2-7 After adding one to six levels of polluting particles according to ISO4406 [26].

Track 8 The hydraulic system is choked.

Figure. 4 shows the MUSIC estimation of pseudo-spectrum of the sound records by first, second and third cellphones, respectively. Observing the curves conclude the following points:

- The lower bands of pseudo-spectrum curves for the clean stage of the hydraulic system, one level and two levels of particle pollution contain similar power as shown by green dashed boxes.

- As pollution reaches the third level, the lower band energy (LBE) of pseudospectrum considerably decreases as shown by blue dashed boxes.

- The LBE remains relatively steady by addition of the polluter particles after the third level.

- By being choked, the LBE of pseudo-spectrum increases almost twice (orange dashed boxes).

In order to clarify and deploy the above observations as a feature, the average energy of the lower-band of each spectrum is obtained as shown in Fig. 5. The resultant LBE of all three phones are averaged and shown in the Fig. 6-up. Namely, as it is observed, LBE remains same as indicated by the ratio of 1 at the first two levels of the pollution. For the higher pollution levels, LBE drops by $27.86 \%$ in average. As per system is choked, the LBE increase to $58.17 \%$ of its value at the clean stage. In brief, LBE drops to almost one third due to pollution, and as the hydraulic system is choked it becomes twice. 


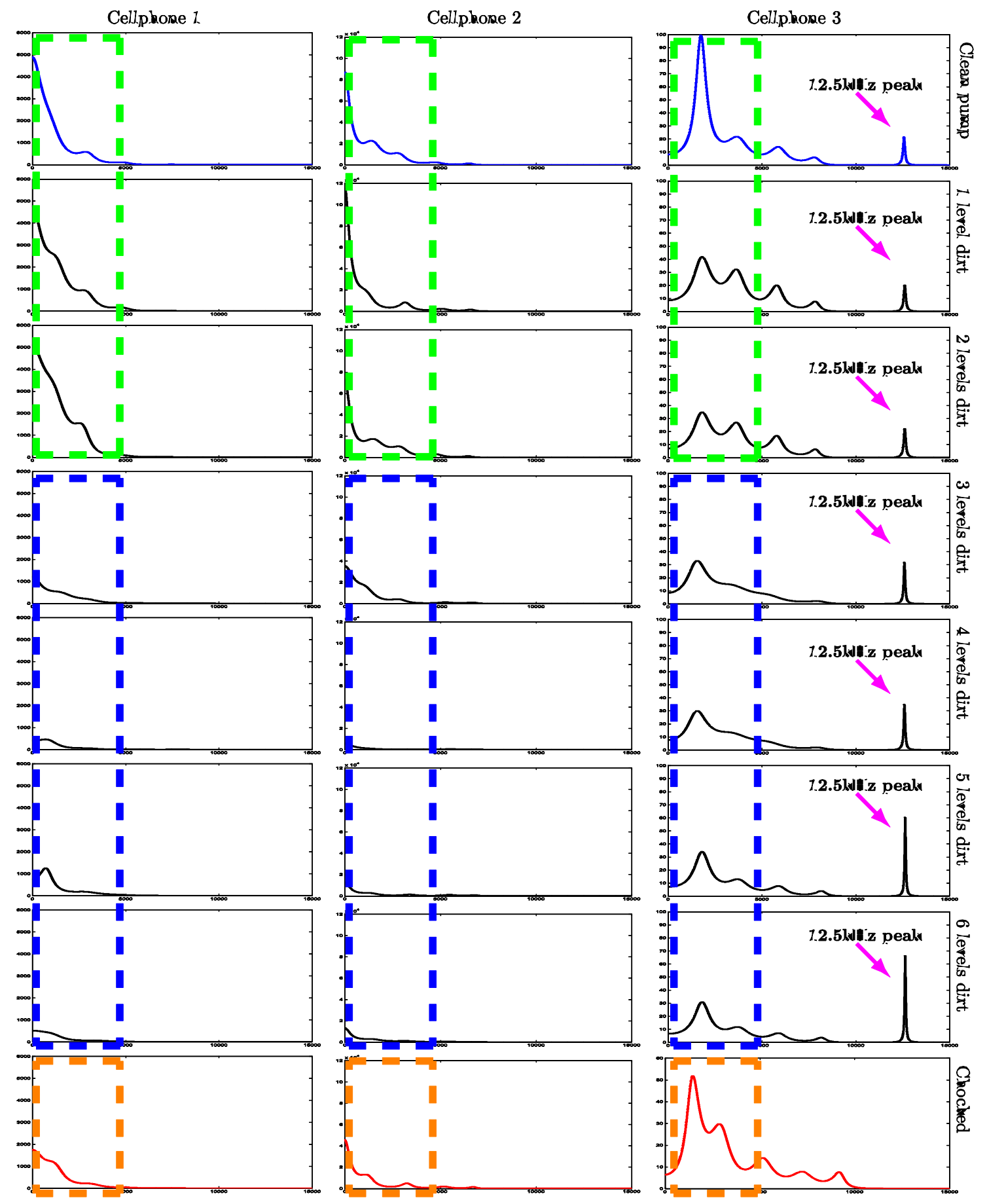

Figure 4. Pseudo-spectrum of the acoustic measurements by three smartphones. At each, the most top curve: the clean hydraulic system, the middle curves: increasing levels of polluting particles, and the most bottom: the chocked hydraulic system. 


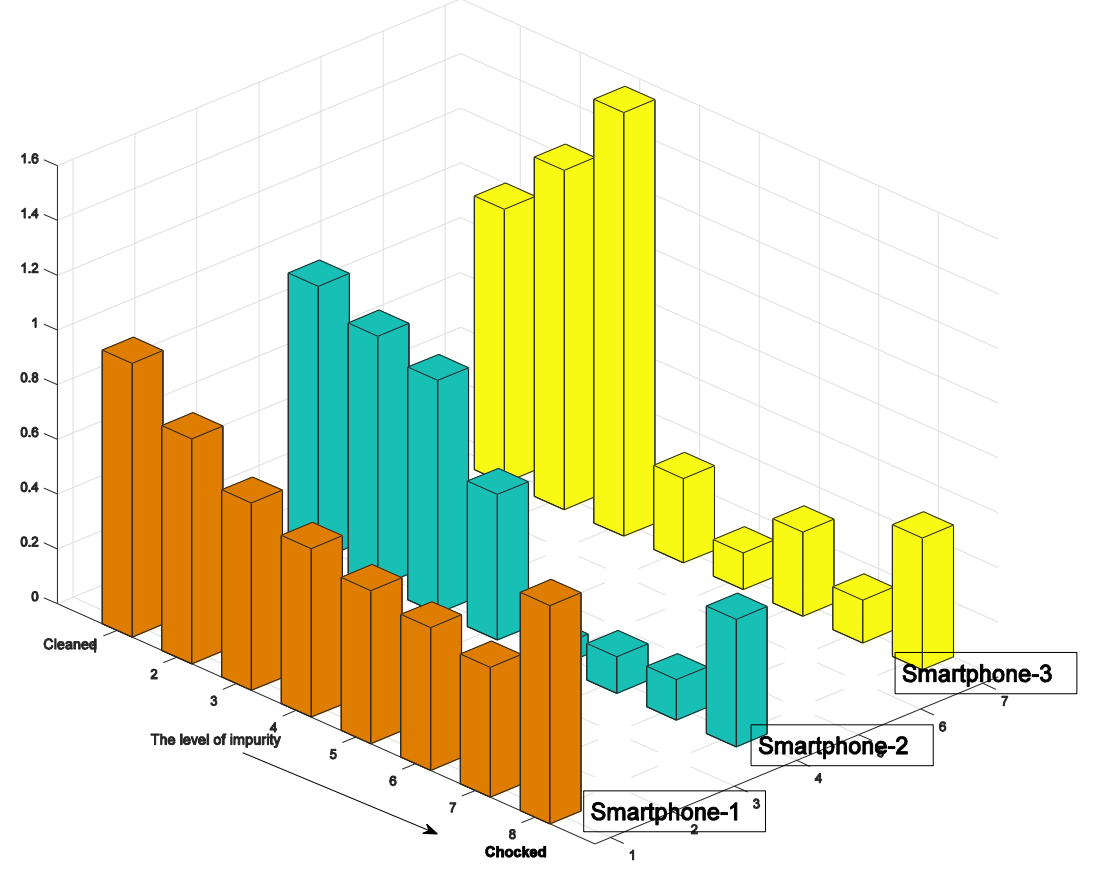

Figure 5. The pseudo-spectrum energy of low band section at each stage to the pollution-free stage for the soundtracks recorded by cell phones 1,2 and 3 in different stages of pollution.

By observing the pseudo-spectrum curves of the third mobile phone records, in addition to the points mentioned above, another point strongly shows off; a pseudospectrum sharp peak in higher frequencies. It has been shown on the figure by a red arrow. It is around one-third the maximum point of the spectrum.

This feature is not observable in the records obtained by the two other phones due to the possible phone integrated anti-noise filters or different qualities of the microphones. The amplitude of the $12.5 \mathrm{kHz}$ peak reaches to three times the highest frequency content of pseudo-spectrum at the third level of dust. As pollutive particles increase, the peak starts to grow up. As the hydraulic system is choked, the peak disappears. We have measured the $12.5 \mathrm{kHz}$ relative peak energy (12.5RPE) in the range of $12-13 \mathrm{kHz}$ with respect to the clean stage as shown in the Figure 6-bottom. The 12.5RPE can be used as the second indicator of the clogging stages in the hydraulic system where its continues increment indicates the relative increase of the particles pollution. As 12.5RPE reaches close to 2, it predicts soon being choked. Its drop to zero indicates already happened choked hydraulic system. From the presented results, we noticed that we need a more rigors experiment and it will be useful to separately recording environmental noise, to subtract it from the records and improving the quality of the features. 

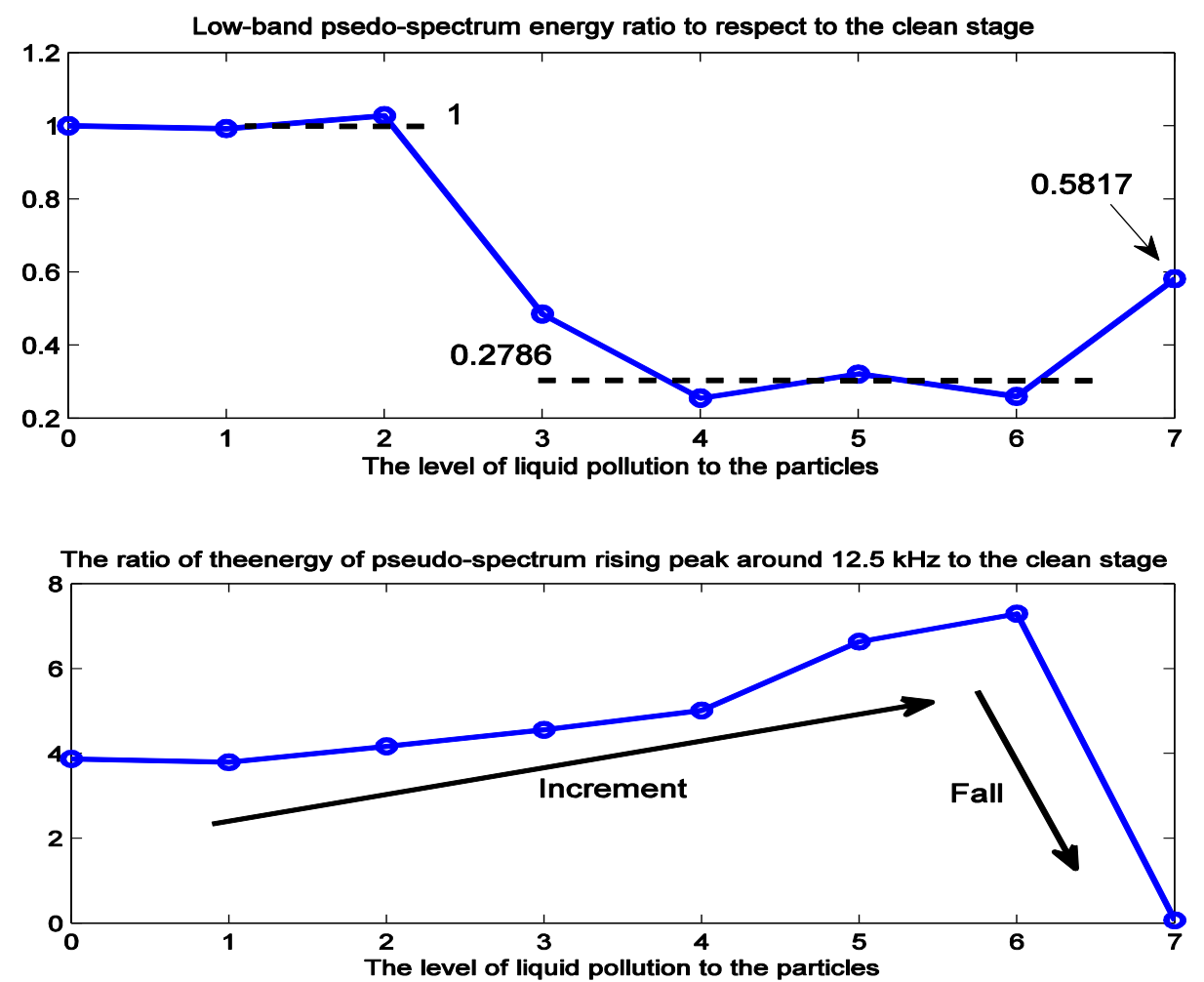

Figure 6. Up: The averaged ratio results of the cell phones 1,2 and 3 of pseudo-spectrum energy of low band section at each stage to the pollution-free stage. Bottom: The ratio of the energy of pseudo-spectrum rising peak around $12.5 \mathrm{KHz}$ to the clean stage.

\section{CONCLUSION}

The fluid pollution monitoring of the Agricultural Machinery hydraulic system, prediction and detection of its choked stage by a user-friendly oil filter sound analyzer smartphone application is a crucial and economic approach. Here, oil filter sound records by three popular brands of cell phones are inspected by pseudo-spectrum analysis. The records are related to eight various conditions: (track 1) clean fluid and new oil filter, (tracks 2-7) one to six levels of particle pollution according to the ISO4406 standard and (track 8 ) choked condition of the hydraulic system. The pseudo spectrum of the normal condition and low levels of dust is almost the same. The MUSIC pseudospectrum analysis of soundtracks leads to two practical features; (i) lower band energy (LBE) of pseudo-spectrum, (ii) $12.5 \mathrm{kHz}$ relative peak energy (12.5RPE) to the clean stage. LBE feature is observable on all three phones. It indicates that as pollution goes further than a level, the LBE drops to around one-third of the clean stage value, and as the hydraulic system is choked, it increases to two third. At the middle of the way to being clogged the power of the pseudo-spectrum considerably reduces, and by being clogged it increases. 12.5RPE is just observed in the third cellphone which is benefited by a wider bandpass of both the microphone and integrated filter. It indicates as the particle pollution gradually increases, the 12.5RPE shows its stepwise increment. As per it reaches to close to twice the clean stage, the choked condition is predicted as next early stage wherein 12.5RPE drops to zero by choked occurrence. Pseudospectrum power besides the proportion of $13 \mathrm{kHZ}$ peak to the maximum low-frequency peak is proposed as an index for fault diagnosis and dust level detection in the agriculture machinery hydraulic system. 


\section{REFERENCES}

1. Prabakar C, Sita Devi K, Selvam S. Labour scarcity - Its immensity and impact on agriculture. Agricul Econom Res Rev 2011;24:373-80.

2. fao.org, Food and Agriculture Organization of the United Nations [Internet]. c2018 [cited 2018 Jul 17]. Available from: http://www.fao.org/3/a-i6887e.pdf

3. Smith BW. How governments can promote automated driving. NML Rev. 2017:47-99.

4. Reese H. Autonomus Driving Levles 0 to 5: Understanding the Differences [Internet]. Innovation [cited 2016 Jan 20] https://www.techrepublic.com/article/autonomous-drivinglevels-0-to-5-understanding-the-differences/.

5. Gebraeel N, Lawley M, Liu R, Parmeshwaran V. Residual life predictions from vibrationbased degradation signals: a neural network approach. IEEE Trans indust electron. 2004;51(3):694-700.

6. Zhao F, Tian Z, Zeng Y. Uncertainty quantification in gear remaining useful life prediction through an integrated prognostics method. IEEE Transactions on Reliability. 2013;62(1):146-59.

7. Scanlon P, Kavanagh DF, Boland FM. Residual life prediction of rotating machines using acoustic noise signals. IEEE Trans. Instrum Measur 2013;62(1):95-108.

8. Gao Z, Cecati C, Ding SX. A survey of fault diagnosis and fault-tolerant techniques-Part I: Fault diagnosis with model-based and signal-based approaches. IEEE Trans Indust Electron. 2015;62(6):3757-67.

9. Damen Technical Agencies. Hydraulic Filtration [Internet] c2018 [cited 2018 Jul 17]. Available from: https://dta.eu/hydraulics/hydraulic-filtration.

10. Day MJ. Condition monitoring of fluid systems-the complete approach. Insight. 1997;39(8):540-7.

11. Hong JH, Day M. Energy Efficiency \& Sustainability the Cleanliness Management Role of Components and System in Automotive and Hydraulics. J Driv \& Cont. 2012;9(4):19-25.

12. Hong JH, Day M. Monitoring of Cleanliness Level in Hydraulic Systems: Obtaining Reliable On-Line data. J of Driv and Cont. 2012;9(2);28-38.

13. Michael PW, Wanke TS, McCambridge MA. Additive and base oil effects in automatic particle counters. J ASTM Inter. 2007;4(4):1-7.

14. Chenghu Z, Haiyan W, Dexing S. Design Principle of Hydraulic and Continuous Filter Regeneration Equipment. Int Conf Measur Tech \& Mech Autom. 2011:1022-5.

15. ISO 11171: Hydraulic fluid power Calibration of automatic particle counters for liquids. International Standards Organization, Switzerland.

16. Wien K. New High-Tech Farm Equipment Is a Nightmare for Farmers [Internet]. Business [cited 2015 Feb 15]. Available from: https://www.wired.com/2015/02/new-high-tech-farmequipment-nightmare-farmers.

17. Sedaaghi MH, Khosravy M. Morphological ECG signal preprocessing with more efficient baseline drift removal. In: Proceedings of the 7th IASTED International Conference; 2003; Canada: ASC; 2003. p. 205-9.

18. Khosravy M, Sedaaghi MH. Impulsive noise suppression of electrocardiogram signals with mediated morphological filters. In: 11th Iranian Conference on Biomedical Engineering; 2004; Iran. 2004. p. 207-12.

19. Khosravy M, Asharif MR, Sedaaghi MH. Morphological adult and fetal ECG preprocessing: employing mediated morphology. IEICE Tech Report. 2008;107(461):363-9.

20. Sedaaghi MH, Daj R, Khosravi M. Mediated morphological filters. In: Proceedings 2001 International Conference on Image Processing; 2001;Thessaloniki Greece; IEEE; 2001. p. 692-5.

21. Khosravy M, Asharif MR, Sedaaghi MH. Medical image noise suppression: using mediated morphology. IEICE Tech Report. 2008;107(461):265-70.

22. Khosravy M, Gupta N, Marina N, Asharif MR, Asharif F, Sethi IK. Blind components processing a novel approach to array signal processing: A research orientation. In: 2015 
International Conference on Intelligent Informatics and Biomedical Sciences (ICIIBMS); 2015; IEEE; 2015. p. 20-6.

23. Khosravy M, Asharif MR, Yamashita K. A theoretical discussion on the foundation of Stone's blind source separation. SIViP. 2011;5:379-88.

24. Khosravy M, Asharif MR, Yamashita K. A PDF-matched short-term linear predictability approach to blind source separation. Int $\mathrm{J}$ Innov Comp, Inform \& Cont. (IJICIC). 2009;5(11):3677-90.

25. Khosravy M, Alsharif MR, Yamashita K. A PDF-Matched Modification to Stone's Measure of Predictability for Blind Source Separation. In: International Symposium on Neural Networks; Springer. 2009. p. 219-28.

26. Khosravy M, Asharif MR, Yamashita K. A Probabilistic Short-length Linear Predictability Approach to Blind Source Separation. In: 23rd International Technical Conference on Circuits/Systems, Computers and Communications (ITC-CSCC 2008); Yamaguchi (Japan); 2008. p. 381-4.

27. Gutierrez CE, Alsharif MR, Cuiwei H, Khosravy M, Villa R, Yamashita K, Miyagi H. Uncover news dynamic by principal component analysis. ICIC Express Letters. 2013;7(4):1245-50.

28. Sedaaghi $\mathrm{MH}$, Daj R, daj Khosravi M. Mediated morphological filters. In: International Conference on Image Processing; 2001; IEEE ICIP; 2001. p. 692-5.

29. ISO 4406: Hydraulic Fluid Power - Fluids - Method For Coding Level of Contamination by Solid Particles, International Standards Organisation, Geneva, Switzerland, 1999.

30. Rahuman S, Veerappan J, Rajesh RV. Classification of Flying Insects with high performance using improved DTW algorithm based on hidden Markov model. Brazilian Archives of Biology and Technology. 2016;59.

31. Shizgal B. Spectral methods in chemistry and physics. In: Scientific Computation. Springer; 2015.

32. Capon J. Maximum-likelihood spectral estimation. In: Nonlinear methods of spectral analysis. Heidelberg (Berlin):Springer; 1979. p. 155-79.

33. Burg JP. The relationship between maximum entropy spectra and maximum likelihood spectra. Geophysics. 1972;37(2):375-6.

34. Pisarenko VF. The retrieval of harmonics from a covariance function. Geophysical Journal International. 1973;33(3):347-66.

35. Schmidt R. Multiple emitter location and signal parameter estimation. IEEE transactions on antennas and propagation. 1986;34(3):276-80. 